\title{
Weak Wavefront Solutions of Maxwell's Equations in Conducting Media
}

\author{
Michael Grinfeld1, Pavel Grinfeld ${ }^{2}$ \\ ${ }^{1}$ The U.S. Army Research Laboratory, WMRD, Aberdeen Proving Ground, Harford County, USA \\ ${ }^{2}$ Mathematics Department, Drexel University, Philadelphia, USA \\ Email: michael.greenfield4.civ@army.mil
}

How to cite this paper: Grinfeld, M. and Grinfeld, P. (2022) Weak Wavefront Solutions of Maxwell's Equations in Conducting Media. Journal of Applied Mathematics and Physics, 10, 190-199.

https://doi.org/10.4236/jamp.2022.101014

Received: October 8, 2021

Accepted: January 21, 2022

Published: January 24, 2022

Copyright $\odot 2022$ by author(s) and Scientific Research Publishing Inc. This work is licensed under the Creative Commons Attribution International License (CC BY 4.0).

http://creativecommons.org/licenses/by/4.0/ (c) (i) Open Access

\begin{abstract}
We analyze the propagation of electromagnetic fronts in unbounded electric conductors. Our analysis is based on the Maxwell model of electromagnetism that includes the displacement current and Ohm's law in its simplest forms. A weak electromagnetic front is a propagating interface at which the electromagnetic field remains continuous while its first- and higher-order derivatives experience finite jump discontinuities. Remarkably, analysis of such fronts can be performed autonomously, i.e. strictly in terms of the quantities defined on the front. This property opens the possibility of establishing exact analytical solutions of the exact Maxwell system along with the evolution of the front.
\end{abstract}

\section{Keywords}

Electric Current in Conductors, Irreversible Thermodynamics, Alegra

Verification, Boundary Value Problems, Exact Solution

\section{Introduction}

Maxwell's theory of electromagnetism was initially met with skepticism. However, upon Hertz's experimental verification of some of its key predictions, it began gaining traction and eventually became the dominant model for electricity and magnetism. Subsequently, numerous treatises have been written on Maxwell's equations which represent the analytical crux of the model.

Maxwell's equations are a system of four PDEs. The system admits various classes of exact analytical solutions. However, one common shortcoming of many of the exact solutions, typically found in the form infinite series or integral expressions, is their analytical complexity. As a result of this complexity, exact solutions often offer less insight than the original equations themselves. Thus, a 
class of exact solutions that are free from overwhelming analytical complexity is of great interest. Propagating wavefronts offer an opportunity for such solutions. It is important to note that the value of exact analytical solutions has not diminished since the advent of the computational revolution. On the contrary, their value has increased since they provide a means of testing the validity of numerical methods.

The electromagnetic fields in many of the essential applications of Maxwell's theory are well described by linearized equations. On the other hand, linear analysis is inadequate for many of the important modern applications, such as nonlinear optics. Needless to say, nonlinear problems pose a much greater challenge-analytically as well as numerically-compared to their linear counterparts. Thus, of particular value are solutions to the nonlinear version of the equations.

In this regard, of considerable interest are exact wavefronts, i.e. moving surfaces along which the electromagnetic field experiences discontinuities. This paper is devoted to weak wavefront solutions, where the electromagnetic field itself is continuous while its derivatives are discontinuous. Pioneering analysis of propagating discontinuities can be found in the monographs by Hadamard [1], Levi-Civita [2], Thomas [3], Luneburg [4], Born and Wolfe [5], and Keller [6].

Exact solutions to Maxwell's equations, i.e. solutions found without making approximations of any kind, are of great value since they exhibit the essential features of the system that include various symmetries and conservation laws. These critical features are typically not preserved by approximation procedures, such as linearization. Fortunately, while the nonlinearities inherent in Maxwell's equations pose a considerable challenge in the pursuit of exact solutions, this challenge can be overcome in wavefront solutions.

The key tool in the analysis of wavefronts is compatibility conditions, originally developed by Hadamard [1], Levi-Civita [2], and Thomas [3]. The first systematic approach to compatibility conditions is found Luneburg [4]. Recently, compatibility conditions were employed by the authors of this paper in the analysis of the simple and double layers in potential theory [7], where the interested reader can find further references and historic discussions.

The method of compatibility conditions, though applicable to nonlinear problems, faces its own difficulties and limitations. One of those difficulties stems from the technical complexity of the conditions themselves. As it often happens, tensor calculus proves effective at overcoming numerous of analytical difficulties. We are indebted to Thomas [3] for his crucial contributions to the development of the relevant tensorial methods. The tensorial aspects of the theory of compatibility conditions have been discussed in detail in [8] where the technique received further analytical development.

\section{The Master System of Equations}

We assume that there are no unbalanced charges within the conductor and con- 
sider electric currents $I^{i}$ governed by the Maxwell equations

$$
\begin{gathered}
\nabla_{i} E^{i}=0 \\
\nabla_{i} H^{i}=0 \\
\frac{1}{c} \frac{\partial H^{i}}{\partial t}=-z^{i j k} \nabla_{j} E_{k} \\
\frac{\partial E^{i}}{\partial t}+4 \pi I^{i}=c z^{i j k} \nabla_{j} H_{k},
\end{gathered}
$$

where $c$ is a speed of light, $E^{i}$ is the electric field, $H^{i}$ is the magnetic field, and $I^{i}$ is the electric current. Furthermore, we assume that the electric field $E^{i}$ and electric current $I^{i}$ are connected by Ohm's law

$$
I^{i}=\sigma E^{i}
$$

where $\sigma$ is the electroconductivity constant.

Eliminating the electric current $I^{i}$ in Equations (1.4) and (1.5), we find

$$
\frac{\partial E^{i}}{\partial t}+4 \pi \sigma E^{i}=c z^{i j k} \nabla_{j} H_{k} .
$$

Differentiating Equation (1.6) with respect to $t$ yields

$$
\frac{\partial^{2} E^{i}}{\partial t^{2}}+4 \pi \sigma \frac{\partial E^{i}}{\partial t}-c^{2} \nabla_{k} \nabla^{k} E^{i}=0 .
$$

Similarly, differentiating Equation (1.3) with respect to $t$ yields

$$
\frac{1}{c} \frac{\partial^{2} H^{i}}{\partial t^{2}}=-z^{i j k} \nabla_{j} \frac{\partial E_{k}}{\partial t} .
$$

Therefore, according to Equation (1.6),

$$
\frac{\partial^{2} H^{i}}{\partial t^{2}}+4 \pi \sigma \frac{\partial H^{i}}{\partial t}-c^{2} \nabla^{k} \nabla_{k} H^{i}=0
$$

as implied by the chain of identities

$$
\begin{aligned}
& \frac{\partial E_{k}}{\partial t}+4 \pi \sigma E_{k}=c z_{k . .}^{m n} \nabla_{m} H_{n} \rightarrow \\
& \frac{\partial E_{k}}{\partial t}+4 \pi \sigma E_{k}=c z_{k . .}^{m n} \nabla_{m} H_{n} \rightarrow \\
& \frac{1}{c} \frac{\partial^{2} H^{i}}{\partial t^{2}}=-z^{i j k} \nabla_{j}\left(c z_{k . .}^{m n} \nabla_{m} H_{n}-4 \pi \sigma E_{k}\right) \rightarrow \\
& \frac{1}{c} \frac{\partial^{2} H^{i}}{\partial t^{2}}=-c z^{i j k} z_{k . .}^{m n} \nabla_{j} \nabla_{m} H_{n}+4 \pi \sigma z^{i j k} \nabla_{j} E_{k} \rightarrow \\
& \frac{\partial^{2} H^{i}}{\partial t^{2}}=-c^{2}\left(z^{i m} z^{j n}-z^{i n} z^{j m}\right) \nabla_{j} \nabla_{m} H_{n}-4 \pi \sigma \frac{\partial H^{i}}{\partial t} \rightarrow \\
& \frac{\partial^{2} H^{i}}{\partial t^{2}}+4 \pi \sigma \frac{\partial H^{i}}{\partial t}=-c^{2}\left(z^{i m} z^{j n} \nabla_{j} \nabla_{m} H_{n}-z^{i n} z^{j m} \nabla_{j} \nabla_{m} H_{n}\right) \rightarrow \\
& \frac{\partial^{2} H^{i}}{\partial t^{2}}+4 \pi \sigma \frac{\partial H^{i}}{\partial t}=c^{2} z^{j m} \nabla^{k} \nabla_{k} H^{i}
\end{aligned}
$$

Equations (1.7), (1.9) are sometimes referred to transport equations. 


\section{Compatibility Conditions for Jump Discontinuities of Derivatives}

We assume that there is a smooth weak front $\Sigma$ inside the conductor, at which the fields $E^{i}(z, t), H^{i}(z, t), I^{i}(z, t)$ remain continuous while their first and second spatial and temporal derivatives experience finite jump discontinuities. Let the surface $\Sigma$ be referred to the Gaussian coordinates $\xi^{\alpha}$ and be described by the equations of the surface $z^{i}=z^{i}\left(\xi^{\alpha}, t\right)$. Whenever possible, we will not omit the indices in the function arguments. The tensor $z_{. \alpha}^{i .}(\xi, t)$, defined by the derivatives $z_{. \alpha}^{i .}(\xi, t) \equiv \partial \mathrm{z}^{i}\left(\xi^{\alpha}, t\right) / \partial \xi^{\alpha}$ is known as the shift tensor. We the help of the shift tensor, we are able to define the surface covariant metric tensor $\xi_{\alpha \beta}(\xi, t) \equiv z_{i j}(x(\xi, t)) z_{. \alpha}^{i .}(\xi, t) z_{. \beta}^{j .}(\xi, t)$. The contravariant surface metric tensor $\xi^{\alpha \beta}(\xi, t)$ is defined as the matrix inverse of $\xi_{\alpha \beta}(\xi, t)$. We use the surface metric tensors for juggling surface indices as well as for defining the surface covariant differentiation $\nabla_{\alpha}$. Let $N^{i}(\xi, t)$ be the unit normal field to $\Sigma$, and $b_{\alpha \beta}(\xi, t)$ be the second form of the surface. Let $C(\xi, t)$ be the velocity of the front.

Introduce the jump discontinuity vectors $f_{E}^{i}(\xi, t), f_{H}^{i}(\xi, t), F_{E}^{i}(\xi, t), F_{H}^{i}(\xi, t)$ as follows:

$$
\begin{aligned}
& f_{E}^{i}(\xi, t) \equiv\left[\nabla_{m} E^{i}\right]_{-}^{+} N^{m}, f_{H}^{i}(\xi, t) \equiv\left[\nabla_{m} H^{i}\right]_{-}^{+} N^{m}, \\
& F_{E}^{i}(\xi, t) \equiv\left[\nabla_{n} \nabla_{m} E^{i}\right]_{-}^{+} N^{m} N^{n}, F_{H}^{i}(\xi, t) \equiv\left[\nabla_{n} \nabla_{m} H^{i}\right]_{-}^{+} N^{m} N^{n}
\end{aligned}
$$

With the help of the vectors $f_{E}^{i}(\xi, t), f_{H}^{i}(\xi, t), F_{E}^{i}(\xi, t), F_{H}^{i}(\xi, t)$, we are able to express all components of the first and second derivatives of $E^{i}(z, t), H^{i}(z, t)$ in terms of the temporal and surface derivatives of $f_{E}^{i}(\xi, t), f_{H}^{i}(\xi, t), F_{E}^{i}(\xi, t), F_{H}^{i}(\xi, t)$ along with the geometrical and kinematical characteristics of the front. In particular, for the first derivatives of the electric and magnetic fields the first-order compatibility conditions read

$$
\begin{aligned}
& {\left[\nabla_{k} E^{i}\right]_{-}^{+} N^{m}=N_{k} f_{E}^{i},\left[\frac{\partial E^{i}}{\partial t}\right]_{-}^{+}=-C f_{E}^{i},} \\
& {\left[\nabla_{k} H^{i}\right]_{-}^{+} N^{m}=N_{k} f_{H}^{i},\left[\frac{\partial H^{i}}{\partial t}\right]_{-}^{+}=-C f_{H}^{i}}
\end{aligned}
$$

For the second-order derivatives, we have

$$
\begin{aligned}
& {\left[\nabla_{k} \nabla_{l} E^{i}\right]_{-}^{+}=F_{E}^{i} N_{k} N_{l}+2 N_{(k} z_{l .)}^{. \alpha} \nabla_{\alpha} f_{E}^{i}-f_{E}^{i} b^{\alpha \beta} z_{k \alpha}^{*} z_{l \beta},} \\
& {\left[\nabla_{k} \frac{\partial E^{i}(z, t)}{\partial t}\right]_{-}^{+}=-F_{E}^{i} N_{k} C+\frac{\delta f_{E}^{i}}{\delta t} N_{k}-z_{k .}^{\alpha} \nabla_{\alpha}\left(C f_{E}^{i}\right),} \\
& {\left[\frac{\partial^{2} E^{i}(z, t)}{\partial t^{2}}\right]_{-}^{+}=F_{E}^{i} C^{2}-2 C \frac{\delta f_{E}^{i}}{\delta t}-f_{E}^{i} \frac{\delta C}{\delta t}}
\end{aligned}
$$

and 


$$
\begin{aligned}
& {\left[\nabla_{k} \nabla_{l} H^{i}\right]_{-}^{+}=F_{H}^{i} N_{k} N_{l}+2 N_{(k} z_{l .)}^{\alpha} \nabla_{\alpha} f_{H}^{i}-f_{H}^{i} b^{\alpha \beta} z_{k \alpha}^{*} z_{l \beta}^{*},} \\
& {\left[\nabla_{k} \frac{\partial H^{i}(z, t)}{\partial t}\right]_{-}^{+}=-F_{H}^{i} N_{k} C+\frac{\delta f_{H}^{i}}{\delta t} N_{k}-z_{k .}^{\alpha} \nabla_{\alpha}\left(C f_{H}^{i}\right),} \\
& {\left[\frac{\partial^{2} H^{i}(z, t)}{\partial t^{2}}\right]_{-}^{+}=F_{H}^{i} C^{2}-2 C \frac{\delta f_{H}^{i}}{\delta t}-f_{H}^{i} \frac{\delta C}{\delta t}}
\end{aligned}
$$

In Equations (2.3), (2.4), $\delta / \delta t$ is the symbol of the invariant time-derivative on the moving surface [3] [8] [9].

\section{The Velocity of Weak Electromagnetic Fronts and Jump Discontinuity Vectors}

Calculating the jump discontinuities of Equations $(1.1-1.3,1.6)$ across a weak front, we find

$$
\begin{gathered}
\nabla_{i}\left[E^{i}\right]_{-}^{+}=0 \\
{\left[\nabla_{i} H^{i}\right]_{-}^{+}=0} \\
\frac{1}{c}\left[\frac{\partial H^{i}}{\partial t}\right]_{-}^{+}=-z^{i j k}\left[\nabla_{j} E_{k}\right]_{-}^{+} \\
{\left[\frac{\partial E^{i}}{\partial t}\right]_{-}^{+}=c z^{i j k}\left[\nabla_{j} H_{k}\right]_{-}^{+},}
\end{gathered}
$$

With the help of the first-order compatibility conditions Equation (2.2), we can transform Equations (3.1) - (3.4) as follows:

$$
\begin{gathered}
f_{E}^{i} N_{i}=0 \\
f_{H}^{i} N_{i}=0 \\
\frac{C}{C} f_{H}^{i}-z^{i j k} N_{j} f_{E k}=0 \\
\frac{C}{C} f_{E}^{i}+z^{i j k} N_{j} f_{H k}=0
\end{gathered}
$$

Equations (3.5) - (3.8) directly imply that the three vectors $f_{E}^{i}, f_{H}^{i}, N^{i}$ are pairwise orthogonal. Eliminating the magnetic jump discontinuity vector $f_{H}^{i}$ between Equations (3.7), (3.8) and using Equation (3.6), we arrive at a linear equation with respect to the electric jump discontinuity vector, i.e.

$$
\left(C^{2}-c^{2}\right) f_{E}^{i}=0 .
$$

Equation (3.9) implies that the nonvanishing jump vector $f_{E}^{i}$ can exist only if

$$
C= \pm c .
$$

Meanwhile, Equation (3.10) in concert with Equations (3.5) - (3.8), implies the relationship 


$$
f_{E}^{i} f_{E i}=f_{H}^{i} f_{H i} .
$$

Let us represent vectors $f_{E}^{i}, f_{H}^{i}$ in the form

$$
f_{E}^{i}=\Delta_{E}^{i}(\xi, t) A_{E}(\xi, t), f_{H}^{i}=\Delta_{H}^{i}(\xi, t) A_{H}(\xi, t),
$$

where $\Delta_{E}^{i}, \Delta_{H}^{i}$ are unit vectors known as the directors, and $A_{E}, A_{H}$ are the magnitudes of the jump discontinuity vectors.

Using the decomposition in Equation (3.12), we can rewrite Equation (3.11) in the form

$$
A_{E}^{2}=A_{H}^{2} .
$$

We can arbitrarily choose any orientation of the vector $\Delta_{E}^{i}$ in the plane orthogonal the unit vector $N_{i}$. The associated vector $\Delta_{H}^{i}$ should satisfy the relationship

$$
\Delta_{H i}=Z_{i j k} N^{j} \Delta_{E}^{k}
$$

The components of the normal are given by the equation

$$
N^{i}=Z^{i j k} \Delta_{E j} \Delta_{H k}
$$

We will see later that the choice of the director $\Delta_{E}^{i}$ at $t=0$ dictates the entire evolution as long as the front remains smooth.

\section{Fronts and Rays}

It is intuitively clear that if the position of the weak front is known at $t=0$ then it can be determined for at all later times since we know that its velocity $C= \pm c$. It is true for the initially smooth front, at least, within a finite interval $0 \leq t \leq t_{c}$, where $t_{c}$ is the moment of appearance of caustics at the front [5]. Let $S: z^{i}=z^{i}(\xi, t)$ be the Gaussian equation of the front at time $t$, and let $S^{\circ}: z^{i}=z^{i}(\xi, 0) \equiv z^{i \circ}(\xi)$ at $t=0$. Let $n^{i \circ}(\xi)$ be the field of unit normal vectors of the surface $S^{\circ}(\xi)$. Then the surface $S$ is described by equation of the surface

$$
S: z^{i}(\xi, t)=z^{i \circ}(\xi) \pm c n^{i \circ}(\xi) t,
$$

where we assume that the ambient coordinate system is affine.

If we fix the moment $t$, we can treat the function $z^{i}=z^{i}(\xi, t)$ in Equation (4.1) as the corresponding position of the front at moment $t$. At the same time, if we fix the Gaussian coordinates $\xi^{\alpha}$ of a point on the surface, then, with changing $t$, we get a straight line, called a ray, as shown in Figure 1.

If we choose a certain area $d S^{\circ}(\xi)$ on the initial front and consider all the rays emanating from $d S^{\circ}(\xi)$, we get a tube of rays. The area $d S$, intercepted by the rays emanating from the current surface $S$ differs from $d S^{\circ}(\xi)$ if the initial interface is not flat. When the tube is infinitesimally small, the ratio $J(\xi, t) \equiv d S / d S^{\circ}$, called the ray's divergence, satisfies the equation

$$
\frac{1}{J} \frac{\partial J}{\partial t}=-C b_{\alpha}^{\alpha} .
$$




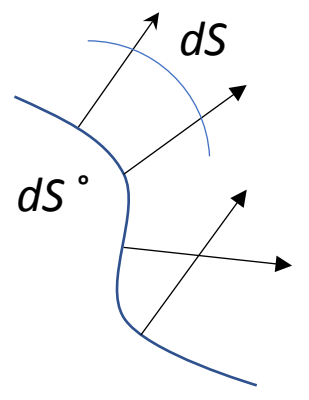

Figure 1. Geometry of rays and front.

Equations (4.1), (4.2) are valid in the case of uniform, isotropic media. Otherwise, they must be replaced with more general relationships [8] [9].

Figure 1 clearly demonstrates that the rays can intersects at $t>0$, beyond which the analysis fails. Extending the analysis beyond this point requires additional physics.

\section{Evolution of the Jump Discontinuity Vectors along Rays}

We now proceed with calculating the jumps discontinuities of the terms in Equation (1.7). We find

$$
\left[\frac{\partial^{2} E^{i}}{\partial t^{2}}\right]_{-}^{+}+4 \pi \sigma\left[\frac{\partial E^{i}}{\partial t}\right]_{-}^{+}-c^{2}\left[\nabla_{k} \nabla^{k} E^{i}\right]_{-}^{+}=0 .
$$

Using the compatibility Equations (2.2), (2.3), we find

$$
F_{E}^{i} C^{2}-2 C \frac{\delta f_{E}^{i}}{\delta t}-f_{E}^{i} \frac{\delta C}{\delta t}-4 \pi \sigma C f_{E}^{i}-c^{2}\left(F_{E}^{i}-f_{E}^{i} b_{\alpha}^{\alpha}\right)=0
$$

If we choose Equation (3.1), then the $\delta / \delta t$-derivative coincides with the partial $\partial / \partial t$-derivative. Using Equation (3.10), we can rewrite Equation (5.2) in the form

$$
2 C \frac{\delta f_{E}^{i}}{\delta t}+f_{E}^{i} \frac{\delta C}{\delta t}+4 \pi \sigma C f_{E}^{i}-c^{2} f_{E}^{i} b_{\alpha}^{\alpha}=0
$$

Using Equation (3.10), we can rewrite Equation (5.3) as follows

$$
2 c \frac{\delta f_{E}^{i}}{\delta t}+4 \pi \sigma C f_{E}^{i}-c^{2} f_{E}^{i} b_{\alpha}^{\alpha}=0
$$

We the help of the decomposition Equation (3.12), we can rewrite Equation (5.4) in the form

$$
\frac{\partial A_{E}}{\partial t} \Delta_{E}^{i}+\frac{\partial \Delta_{E}^{i}}{\partial t} A_{E}+2 \pi \sigma \Delta_{E}^{i} A_{E}-\frac{1}{2} c b_{\alpha}^{\alpha} \Delta_{E}^{i} A_{E}=0 .
$$

Contracting Equation (5.5) with the unit vector $\Delta_{E i}$, we find

$$
\frac{\partial A_{E}}{\partial t}+2 \pi \sigma A_{E}-\frac{1}{2} c b_{\alpha}^{\alpha} A_{E}=0
$$

Using Equation (4.2), we can rewrite Equation (5.6) as 


$$
\frac{\partial}{\partial t}\left(\mathrm{e}^{4 \pi \sigma t} J A_{E}^{2}\right)=0,
$$

as implied by the following chain of identities:

$$
\begin{aligned}
& 2 \frac{\partial A_{E}}{\partial t}+4 \pi \sigma A_{E}+\frac{1}{J} \frac{\partial J}{\partial t} A_{E}=0 \rightarrow \\
& \frac{\partial \ln \left(J A_{E}^{2}\right)}{\partial t}+4 \pi \sigma=0 \rightarrow \\
& \frac{\partial\left(J A_{E}^{2}\right)}{\partial t}+4 \pi \sigma J A_{E}^{2}=0 \rightarrow \\
& \frac{\partial}{\partial t}\left(\mathrm{e}^{4 \pi \sigma t} J A_{E}^{2}\right)=0
\end{aligned}
$$

Contracting Equation (4.5) with unit vector $\Delta_{H i}$, we get

$$
\frac{\partial \Delta_{E}^{i}}{\partial t} \Delta_{H i}=0
$$

Furthermore, differentiating the identity

$$
\Delta_{E}^{i} N_{i}=0, \Delta_{E}^{i} \Delta_{E i}=1
$$

yields

$$
\frac{\partial \Delta_{E}^{i}}{\partial t} \Delta_{H i}=0, \frac{\partial \Delta_{E}^{i}}{\partial t} \Delta_{E i}=0 .
$$

Summarizing, we arrive at the relationship

$$
\frac{\partial \Delta_{E}^{i}}{\partial t}=0
$$

In view of Equation (5.8), the directors $\Delta_{E}^{i}$ and $\Delta_{H}^{i}$ remain the same along each of the rays.

\section{Conclusions}

We analyzed the propagation of electromagnetic fronts in an unbounded electric conductor with the isotropic electric conductivity constant $\sigma$. The weak electromagnetic front is a moving surface at which the electric and magnetic fields remain continuous while their first- and higher-order derivatives experience finite jumps. We demonstrated that all of the discontinuity jumps across the weak front can be expressed in terms of the jump discontinuity vectors along with their derivatives with respect to time and the surface coordinates. In particular, the jumps of all or the first- and second-order derivatives can be expressed in terms of the discontinuity vectors $f_{E}^{i}(\xi, t), f_{H}^{i}(\xi, t), F_{E}^{i}(\xi, t), F_{H}^{i}(\xi, t)$ defined by Equation (2.1). The jump discontinuities of the first-order derivatives are given in Equation (2.2), while the jump discontinuities of the second-order derivatives are given by the second-order compatibility conditions in Equation (2.3).

Remarkably, the evolution of the fields $f_{E}^{i}(\xi, t), f_{H}^{i}(\xi, t), F_{E}^{i}(\xi, t), F_{H}^{i}(\xi, t)$ can be separated from the analysis of the electric field $E^{i}$ and magnetic field 
$H^{i}$ in the domains of smoothness. In this sense, the analysis of weak fronts appears to be autonomous. This autonomous character of the weak fronts appears to have tremendous utility since the bulk equation contains four independent variables, whereas the autonomous system only three.

Applying the first-order compatibility Equations (2.2) to the Maxwell system, (1.1) - (1.4) along with Ohm's law Equation (1.5), we arrived at the linear homogeneous system (3.5) - (3.8). Analysis of Equations (3.5) - (3.8) directly implies that 1) the velocity $C$ of the weak front is equal to $\pm c$, where $c$ is the speed of light in vacuum; 2) The three vectors $f_{E}^{i}, f_{H}^{i}, N^{i}$ are pairwise orthogonal and satisfy the relationship $f_{E}^{i} f_{E i}=f_{H}^{i} f_{H i}$ captured in Equation (3.11), 3) the velocity $C$ of the weak front does not depend on the electroconductivity $\sigma$.

The position of the weak front $S: z^{i}(\xi, t)$ is defined in an affine coordinate system by the Equation (4.1): $z^{i}(\xi, t)=z^{i_{0}}(\xi) \pm c n^{i_{0}}(\xi) t$, where $S^{\circ}: z^{i}=z^{i}(\xi, 0) \equiv z^{i \circ}(\xi)$ at $t=0$ and $n^{i \circ}(\xi)$ is the field of unit normal vectors of the surface $S^{\circ}$. If we fix the moment of time $t$, then we can treat the function $z^{i}=z^{i}\left(\xi^{\alpha}, t\right)$ in Equation (4.1) as the corresponding position of the front at moment $t$. At the same time, if we fix a pair of the Gaussian coordinates $\xi^{\alpha}$ then with changing $t$, we get a straight line, called a ray, as shown in Figure 1. Consequently, $z^{i}(\xi, t)=z^{i^{i}}(\xi) \pm c n^{i \circ}(\xi) t$ delivers the Gaussian equation of the weak front in the special "ray" coordinates.

Applying the second-order compatibility Equations (2.4) to the transport Equations (1.7), (1.9), we establish our main result $\partial\left(\mathrm{e}^{4 \pi \sigma t} J A_{E}^{2}\right) / \partial t=0$ in Equation (5.9). This equation describes how the amplitude of the jump discontinuity changes along the ray. It shows that the jump intensity grows when the ray divergence decays and vice versa. Furthermore, there is an additional mechanism of exponential decay due to Ohm's resistance dissipation. Finally, Equation (5.12) shows that the director vector $\Delta_{E}^{i}$ remains constant along the ray.

Ray constructions also appear when considering high frequency asymptotics [4] [5]. However, the method of weak fronts is equally applicable in nonlinear problems, and it is exact rather than asymptotic.

\section{Conflicts of Interest}

The authors declare no conflicts of interest regarding the publication of this paper.

\section{References}

[1] Hadamard, J. (1903) Leçons sur la Propagation des Ondes et les Équations de l'Hydrodynamique. Hermann.

[2] Levi-Civita, T. (1931) Caratteristiche dei sistemi differenziali e propagazione ondosa. Bologna.

[3] Thomas, T.Y. (1958) Plastic Flow and Fracture in Solids. Indiana University Mathematics Journal, 7, 291-322. https://doi.org/10.1512/iumj.1958.7.57021

[4] Luneburg, R.K. (1964) Mathematical Theory of Optics. University of California Press, Berkeley, California. https://doi.org/10.1525/9780520328266 
[5] Born, M. and Wolfe, E. (1999) Principles of Optics. Cambridge University Press, Cambridge. https://doi.org/10.1017/CBO9781139644181

[6] Keller, J.B. (1962) Geometrical Theory of Diffraction. Journal of the Optical Society of America, 52, 116-130. https://doi.org/10.1364/JOSA.52.000116

[7] Grinfeld, M.A. and Grinfeld, P.M. (2021) Second-Order Discontinuities for Simple and Double Layers. Journal of Applied Mathematics and Physics, 9, No. 9. https://doi.org/10.4236/jamp.2021.99140

[8] Grinfeld, P.M. (2014) Introduction to Tensor Analysis and the Calculus of Moving Surfaces. Springer, Heidelberg, Berlin. https://doi.org/10.1007/978-1-4614-7867-6

[9] Grinfeld, M.A. (1991) Thermodynamic Methods in the Theory of Heterogeneous Systems. 